\title{
Effects of Environmental Exposure to Cadmium and Lead on the Risks of Diabetes and Kidney Dysfunction
}

\author{
Supabhorn Yimthiang ${ }^{1}\left(\mathbb{D}\right.$, Phisit Pouyfung ${ }^{1}$, Tanaporn Khamphaya ${ }^{1}\left({ }^{\infty}\right.$, Saruda Kuraeiad ${ }^{2}{ }^{(D}$, \\ Paleeratana Wongrith ${ }^{3}$, David A. Vesey ${ }^{4,5}$, Glenda C. Gobe ${ }^{4,6,7}$ and $^{\circ}$ and Soingwan Satarug ${ }^{4, *}$
}

1 Occupational Health and Safety, School of Public Health, Walailak University, Nakhon Si Thammarat 80160, Thailand; ksupapor@mail.wu.ac.th (S.Y.); phisit.po@mail.wu.ac.th (P.P.); tanaporn.kh@mail.wu.ac.th (T.K.)

2 Medical Technology, School of Allied Health Sciences, Walailak University, Nakhon Si Thammarat 80160, Thailand; saruda.ku@wu.ac.th

3 Community Public Health, School of Public Health, Walailak University, Nakhon Si Thammarat 80160, Thailand; paleeratana.wo@wu.ac.th

4 Kidney Disease Research Collaborative, Centre for Health Services Research, The University of Queensland Translational Research Institute, Brisbane 4102, Australia; david.vesey@health.qld.gov.au (D.A.V.); g.gobe@uq.edu.au (G.C.G.)

5 Department of Nephrology, Princess Alexandra Hospital, Brisbane 4075, Australia

6 School of Biomedical Sciences, The University of Queensland, Brisbane 4072, Australia

7 NHMRC Centre of Research Excellence for CKD, QLD, UQ Health Sciences, Royal Brisbane, and Women's Hospital, Brisbane 4029, Australia

* Correspondence: sj.satarug@yahoo.com.au

\section{check for} updates

Citation: Yimthiang, S.; Pouyfung, P.; Khamphaya, T.; Kuraeiad, S.;

Wongrith, P.; Vesey, D.A.; Gobe, G.C.; Satarug, S. Effects of Environmental Exposure to Cadmium and Lead on the Risks of Diabetes and Kidney Dysfunction. Int. J. Environ. Res. Public Health 2022, 19, 2259. https:/ / doi.org/10.3390/ijerph19042259

Academic Editors: Yasushi Suwazono and Masaru Sakurai

Received: 13 January 2022

Accepted: 15 February 2022

Published: 16 February 2022

Publisher's Note: MDPI stays neutral with regard to jurisdictional claims in published maps and institutional affiliations.

Copyright: (C) 2022 by the authors. Licensee MDPI, Basel, Switzerland. This article is an open access article distributed under the terms and conditions of the Creative Commons Attribution (CC BY) license (https:// creativecommons.org/licenses/by/ $4.0 /)$.

\begin{abstract}
Environmental exposure to cadmium $(\mathrm{Cd})$ or lead $(\mathrm{Pb})$ is independently associated with increased risks of type 2 diabetes, and chronic kidney disease. The aim of this study was to examine the effects of concurrent exposure to these toxic metals on the risks of diabetes and kidney functional impairment. The $\mathrm{Cd}$ and $\mathrm{Pb}$ exposure levels among study subjects were low to moderate, evident from the means for blood concentrations of $\mathrm{Cd}$ and $\mathrm{Pb}\left([\mathrm{Cd}]_{\mathrm{b}}\right.$ and $\left.[\mathrm{Pb}]_{\mathrm{b}}\right)$ of $0.59 \mu \mathrm{g} / \mathrm{L}$ and $4.67 \mu \mathrm{g} / \mathrm{dL}$, respectively. Of 176 study subjects (mean age 60), 71 (40.3\%) had abnormally high fasting plasma glucose levels. Based on their $[\mathrm{Cd}]_{\mathrm{b}}$ and $[\mathrm{Pb}]_{\mathrm{b}}, 53,71$, and 52 subjects were assigned to $\mathrm{Cd}$ and $\mathrm{Pb}$ exposure profiles 1, 2, and 3, respectively. The diagnosis of diabetes was increased by 4.2 -fold in those with an exposure profile $3(p=0.002)$, and by 2.9 -fold in those with the estimated glomerular filtration $(\mathrm{eGFR}) \leq 60 \mathrm{~mL} / \mathrm{min} / 1.73 \mathrm{~m}^{2}(p=0.029)$. The prevalence odds ratio (POR) for albuminuria was increased by 5-fold in those with plasma glucose levels above kidney threshold of $180 \mathrm{mg} / \mathrm{dL}$ ( $p=0.014)$, and by 3.1-fold in those with low eGFR) $(p=0.050)$. Collectively, these findings suggest that the $\mathrm{Cd}$ and $\mathrm{Pb}$ exposure profiles equally impact kidney function and diabetes risk.
\end{abstract}

Keywords: cadmium; lead; albuminuria; diabetes; fasting plasma glucose; glomerular filtration rate

\section{Introduction}

Chronic environmental exposure to cadmium $(\mathrm{Cd})$ has been associated with increased risks of prediabetes and diabetes in the general populations in the U.S. [1-4], Korea [5], and China [6]. Two Norwegian population studies reported exposure to $\mathrm{Cd}$ and lead $(\mathrm{Pb})$ as potential risk factors for diabetes $[7,8]$. Low environmental exposure to $\mathrm{Cd}$ has been identified as a risk factor for chronic kidney disease (CKD) in cross-sectional studies in Spain [9], Korea [10], and the U.S. [11-15]. The diagnosis of CKD is based on albumin-tocreatinine ratio (ACR) above $30 \mathrm{mg} / \mathrm{g}$ creatinine (albuminuria) and/or a decrease in the glomerular filtration rate (GFR) to $60 \mathrm{~mL} / \mathrm{min} / 1.73 \mathrm{~m}^{2}$ (low eGFR) that persists for at least three months [16-19]. In the Dallas lead project, an elevation of blood $\mathrm{Pb}\left([\mathrm{Pb}]_{\mathrm{b}}\right)$ was associated with a marked reduction in eGFR among residents of a lead smelter community [20]. 
In prospective cohort studies in Sweden, low environmental $\mathrm{Pb}$ exposure was linked to a GFR decrease, CKD onset, and end-stage kidney disease (ESKD) [21,22].

Simultaneous exposure to $\mathrm{Cd}$ and $\mathrm{Pb}$ is commonly encountered by the general populations of various nations [23,24], including the U.S. [25-28], Canada [29], Taiwan [30], and Korea [31]. In the U.S. National Health and Nutrition Examination Surveys (NHANES) 2007-2012, half of the participants, aged $\geq 6$ years had $[\mathrm{Cd}]_{\mathrm{b}}$ and $[\mathrm{Pb}]_{\mathrm{b}}$ or urinary excretion rates of $\mathrm{Cd}$ and $\mathrm{Pb}$ above population median levels [27]. It was noted in the Cadmibel study that people with diabetes were more susceptible than those with no diabetes to $\mathrm{Cd}$ toxicity in kidneys [32]. A similar observation was subsequently made in women from Sweden [33,34], the Torres Strait, Australia [35], Korea [36], and the U.S. [13].

The aim of this study was to investigate the effects of concurrent exposure to $\mathrm{Cd}$ and $\mathrm{Pb}$ in 176 Thai subjects of which 88 were diagnosed diabetes, and 88 were apparently healthy non-diabetics. $[\mathrm{Cd}]_{b}$ and $[\mathrm{Pb}]_{b}$ levels were used as exposure estimates, while estimated GFR (eGFR) and albumin-to-creatinine ratio (ACR) served as kidney functional measures. GFR is considered to indicate function of surviving nephrons [16-18]. In practice, the GFR is estimated from equations, including the Chronic Kidney Disease Epidemiology Collaboration (CKD-EPI) [17], and is reported as an estimated GFR (eGFR). ACR is primarily a function of glomerular membrane permeability, and kidney proximal tubular reabsorption [37].

\section{Materials and Methods}

\subsection{Participants}

This study used a purposive sampling method to recruit type 2 diabetic subjects together with age- and gender-matched non-diabetic controls of equal number from a local health center of Pakpoon Municipality, Nakhon Si Thammarat Province, Thailand. It was undertaken during June 2020 to May 2021. The inclusion criteria were residents of Pakpoon municipality, 40 years of age or older who were diagnosed with type 2 diabetes or were apparently healthy. The exclusion criteria were non-residents of Pakpoon municipality, pregnancy, breast-feeding, a hospital record or physician's diagnosis of an advanced chronic disease. All subjects were provided with details of study objectives, study procedures, benefits, and potential risks, and they all provided their written informed consents prior to participation. The sociodemographic data, education attainment, occupation, health status, family history of diabetes, and smoking status were obtained by structured interview questionnaires. Diabetes was defined as plasma glucose [Glc $]_{p}$ levels $\geq 126 \mathrm{mg} / \mathrm{dL}$ or a physician's diagnosis. Hypertension was defined as systolic blood pressure $\geq 140 \mathrm{mmHg}$, or diastolic blood pressure $\geq 90 \mathrm{mmHg}$. After excluding subjects with missing data, 176 subjects ( 88 with a diabetes diagnosis and 88 apparently healthy, non-diabetic controls) were included in the present study.

\subsection{Simultaneous Blood and Urine Sampling and Biochemical Analysis}

Participants were instructed to fast overnight, and the collection of blood and urine samples was carried out at a local health center of Pakpoon Municipality in the morning of the following day. For glucose assay, blood samples were collected in tubes containing fluoride as an inhibitor of glycolysis. For an analysis of $\mathrm{Cd}$ and $\mathrm{Pb}$, blood samples were collected in separate tubes containing ethylene diamine tetra-acetic acid (EDTA) as an anticoagulant. The blood and urine samples were kept on ice and transported within $1 \mathrm{~h}$ to the medical technology laboratory of Walailak University, where plasma samples were prepared for various biochemical analyses. The remainder plasma and whole blood samples were aliquoted as were urine samples, stored at $-80^{\circ} \mathrm{C}$ for later analysis. Fasting plasma glucose concentrations $\left([\mathrm{Glc}]_{\mathrm{p}}\right.$ ) were measured to ascertain diabetes diagnosis and diabetes free stage of controls. The assay of plasma concentration of glucose was based on colorimetry. Assays of creatinine in urine and plasma $\left.\left([\mathrm{cr}]_{\mathrm{u}},[\mathrm{cr}]_{\mathrm{p}}\right]\right)$ were based on the Jaffe reaction. Urine concentration of albumin ([Alb $]_{\mathrm{u}}$ ) was determined using an immunoturbidimetric method. 


\subsection{Analysis of Blood Concentrations of $\mathrm{Cd}$ and $\mathrm{Pb}$}

Blood concentrations of $\mathrm{Cd}$ and $\mathrm{Pb}\left([\mathrm{Cd}]_{b},[\mathrm{~Pb}]_{b}\right)$ were determined with the GBC System 5000 Graphite Furnace Atomic Absorption Spectrophotometer (GBC Scientific Equipment, Hampshire, IL, USA). Multielement standards were used to calibrate metal analysis (Merck KGaA, Darmstadt, Germany). Reference urine and whole blood metal control levels 1, 2, and 3 (Lyphocheck, Bio-Rad, Hercules, CA, USA) were used for quality control, analytical accuracy, and precision assurance. The analytical accuracy of metal detection was checked by an external quality assessment every 3 years. All test tubes, bottles, and pipettes used in metal analysis were acid-washed and rinsed thoroughly with deionized water. When $[\mathrm{Cd}]_{b}$ and $[\mathrm{Pb}]_{b}$ levels were less than their detection limits, the concentrations assigned were their detection limits divided by the square root of 2 [38]. Ninety-four subjects $(53.4 \%)$ had $[\mathrm{Pb}]_{\mathrm{b}}$ below the detection limit of $3 \mu \mathrm{g} / \mathrm{dL}$, and $61(34.6 \%)$ had $[\mathrm{Cd}]_{\mathrm{b}}$ below the detection limit of $0.1 \mu \mathrm{g} / \mathrm{L}$.

\subsection{Toxic Metal Exposure Profiling}

To enable evaluation of the impacts of simultaneous exposure to $\mathrm{Cd}$ and $\mathrm{Pb}$ on the risks of diabetes and kidney dysfunction, exposure to these toxic metals was based on $[\mathrm{Cd}]_{\mathrm{b}}$ and $[\mathrm{Pb}]_{\mathrm{b}}$. Each subject was assigned to the exposure profile 1, 2, or 3 by comparing her / his $[\mathrm{Cd}]_{b}$ and $[\mathrm{Pb}]_{\mathrm{b}}$ with the median for $[\mathrm{Cd}]_{\mathrm{b}}$ of $0.3 \mu \mathrm{g} / \mathrm{L}$ and the median for $[\mathrm{Pb}]_{\mathrm{b}}$ of $2.12 \mu \mathrm{g} / \mathrm{dL}$. Given the sample size of 176, we used the median as a cutoff value to obtain subgroups with sufficient numbers of participants. Exposure profile 1 was defined as $[\mathrm{Cd}]_{\mathrm{b}}$ and $[\mathrm{Pb}]_{\mathrm{b}} \leq$ medians. Exposure profile 2 was defined as $[\mathrm{Cd}]_{\mathrm{b}}$ or $[\mathrm{Pb}]_{\mathrm{b}}$ levels $\geq$ medians Exposure profile 3 was defined as $[\mathrm{Cd}]_{\mathrm{b}}$ and $[\mathrm{Pb}]_{\mathrm{b}}>$ medians. There were 53,71 , and 52 subjects with exposure profiles 1,2 , and 3 , respectively.

\subsection{Estimated Glomerular Filtration Rates (eGFR)}

The glomerular filtration rate was estimated with CKD-EPI equations [17], which were validated by inulin clearance [17]. Male eGFR $=141 \times$ [serum creatinine/0.9] $^{\mathrm{Y}}$ $\times 0.993^{\text {age }}$, where $\mathrm{Y}=-0.411$ if serum creatinine $\leq 0.9 \mathrm{mg} / \mathrm{dL}, \mathrm{Y}=-1.209$ if serum creatinine $>0.9 \mathrm{mg} / \mathrm{dL}$. Female $\mathrm{eGFR}=144 \times[\text { serum creatinine } / 0.7]^{\mathrm{Y}} \times 0.993^{\text {age }}$, where $\mathrm{Y}=-0.329$ if serum creatinine $\leq 0.7 \mathrm{mg} / \mathrm{dL}, \mathrm{Y}=-1.209$ if serum creatinine $>0.7 \mathrm{mg} / \mathrm{dL}$. For dichotomous comparisons, CKD was defined as eGFR $\leq 60 \mathrm{~mL} / \mathrm{min} / 1.73 \mathrm{~m}^{2}$ and CKD stages 1 , 2, 3a, 3b, 4, and 5 corresponded to eGFR of 90-119, 60-89, 45-59, 30-44, 15-29, and $<15 \mathrm{~mL} / \mathrm{min} / 1.73 \mathrm{~m}^{2}$, respectively.

\subsection{Statistical Analysis}

Data were analyzed with IBM SPSS Statistics 21 (IBM Inc., New York, NY, USA). The Kruskal-Wallis test was used to assess differences in means among three exposure groups, and the Pearson chi-squared test was used to assess differences in percentages. The one-sample Kolmogorov-Smirnov test was used to identify departures of continuous variables from a normal distribution, and a base- 10 logarithmic transformation was applied to variables that showed rightward skewing before they were subjected to parametric statistical analysis. The multivariable logistic regression analysis was used to determine the Prevalence Odds Ratio (POR) for dichotomized outcomes. Abnormal fasting plasma glucose was defined as [Glc] $]_{p} \geq 110 \mathrm{mg} / \mathrm{dL}$. Diabetes was diagnosed when fasting [Glc] were $\geq 126 \mathrm{mg} / \mathrm{dL}$. The renal threshold for glucose was assumed to be [Glc $]_{\mathrm{p}} \geq 180 \mathrm{mg} / \mathrm{dL}$. Albuminuria was defined as a ACR $\geq 20 \mathrm{mg} / \mathrm{g}$ for men and $\geq 30 \mathrm{mg} / \mathrm{g}$ for women. The Pearson's correlation analysis was used to assess the strength of correlation of [Glc $]_{p}$ and other variables. The means for $[\mathrm{Glc}]_{p}$ adjusted for age, BMI, and interaction in groups of subjects were obtained by univariate/covariance analysis with Bonferroni correction in multiple comparisons. For all tests, $p$-values $\leq 0.05$ were considered to indicate statistical significance. 


\section{Results}

\subsection{Characteristics of Participants}

Among 176 participants, half were given type 2 diabetes diagnosis by their medical records, while 3 of 88 persons recruited as non-diabetic controls had fasting plasma glucose $[\mathrm{Glc}]_{\mathrm{p}} \geq 126 \mathrm{mg} / \mathrm{dL}$, a diabetes diagnosis level (Table 1 ).

Table 1. Characteristics of participants grouped by their cadmium and lead exposure profiles.

\begin{tabular}{|c|c|c|c|c|c|}
\hline \multirow{2}{*}{ Variables } & \multirow{2}{*}{$\begin{array}{l}\text { All Participants } \\
\qquad n=176\end{array}$} & \multicolumn{3}{|c|}{ Cadmium and Lead Exposure ${ }^{a}$} & \multirow{2}{*}{$p$} \\
\hline & & Profile $1, n 53$ & Profile $2, n 71$ & Profile $3, n 52$ & \\
\hline Women, \% & 80.7 & 90.6 & 76.1 & 76.9 & 0.092 \\
\hline Diabetes diagnosis $\mathrm{b}, \%$ & 50.0 & 35.8 & 46.5 & 69.2 & 0.002 \\
\hline Diabetes duration, years & $9.3 \pm 7.6$ & $8.8 \pm 7.8$ & $9.2 \pm 7.9$ & $9.5 \pm 7.4$ & 0.910 \\
\hline Smoking, $\%$ & 9.7 & 1.9 & 9.9 & 17.9 & 0.028 \\
\hline Hypertension $\mathrm{c} \%$ & 52.0 & 48.0 & 45.1 & 65.4 & 0.067 \\
\hline SBP, $\mathrm{mmHg}$ & $138 \pm 17$ & $137 \pm 17$ & $136 \pm 15$ & $142 \pm 19$ & 0.122 \\
\hline $\mathrm{DBP}, \mathrm{mmHg}$ & $84 \pm 9.5$ & $83 \pm 9.4$ & $83 \pm 8.7$ & $86 \pm 10.4$ & 0.226 \\
\hline Age, years & $59.9 \pm 9.7$ & $59.5 \pm 10.6$ & $60.8 \pm 9.2$ & $58.9 \pm 9.4$ & 0.639 \\
\hline BMI, $\mathrm{kg} / \mathrm{m}^{2}$ & $25.4 \pm 4.7$ & $25.8 \pm 4.3$ & $25.0 \pm 4.0$ & $25.6 \pm 5.9$ & 0.426 \\
\hline \multicolumn{6}{|l|}{ BMI classification ${ }^{d}$} \\
\hline Thin, $\%$ & 6.8 & 5.7 & 4.2 & 11.5 & 0.472 \\
\hline Normal, \% & 28.4 & 26.4 & 32.4 & 25.0 & 0.162 \\
\hline Overweight, \% & 54.0 & 56.6 & 54.9 & 50.0 & 0.247 \\
\hline Obesity, \% & 10.8 & 11.3 & 8.5 & 13.5 & 0.949 \\
\hline$[\mathrm{cr}]_{\mathrm{p}}, \mathrm{mg} / \mathrm{dL}$ & $0.87 \pm 0.24$ & $0.82 \pm 0.15$ & $0.89 \pm 0.19$ & $0.90 \pm 0.36$ & 0.133 \\
\hline$[\mathrm{cr}]_{\mathrm{u}}, \mathrm{mg} / \mathrm{dL}$ & $89.2 \pm 54.1$ & $92.2 \pm 51.7$ & $90.1 \pm 53.7$ & $84.8 \pm 57.9$ & 0.499 \\
\hline$[\mathrm{Alb}]_{\mathrm{u}}, \mathrm{mg} / \mathrm{dL}$ & $30.9 \pm 79.9$ & $23.8 \pm 64.1$ & $34.4 \pm 90.2$ & $33.8 \pm 81.1$ & 0.564 \\
\hline$[\mathrm{Pb}]_{b}, \mu \mathrm{g} / \mathrm{dL}$ & $4.67 \pm 4.88$ & $2.12 \pm 0.00$ & $4.58 \pm 5.30$ & $7.38 \pm 5.38$ & $<0.001$ \\
\hline$[\mathrm{Cd}]_{b}, \mu \mathrm{g} / \mathrm{L}$ & $0.59 \pm 0.74$ & $0.05 \pm 0.05$ & $0.65 \pm 0.78$ & $1.05 \pm 0.72$ & $<0.001$ \\
\hline $\mathrm{eGFR} \mathrm{e}^{\mathrm{e}}, \mathrm{mL} / \mathrm{min} / 1.73 \mathrm{~m}^{2}$ & $79 \pm 18$ & $81 \pm 16$ & $77 \pm 17$ & $81 \pm 21$ & 0.269 \\
\hline$\leq 60, \%$ & 16.5 & 11.3 & 22.5 & 13.5 & 0.196 \\
\hline $\mathrm{ACR}, \mathrm{mg} / \mathrm{g}$ creatinine & $41.4 \pm 103.8$ & $28.4 \pm 75.8$ & $38.5 \pm 92.3$ & $59.3 \pm 139.2$ & 0.387 \\
\hline$<30, \%$ & 78.4 & 82.7 & 79.1 & 72.9 & 0.155 \\
\hline $30-299, \%$ & 17.4 & 15.4 & 16.4 & 20.8 & 0.786 \\
\hline$\geq 300, \%$ & 4.2 & 1.9 & 4.5 & 6.3 & 0.565 \\
\hline$[\mathrm{Glc}]_{\mathrm{p}}{ }^{\mathrm{f}} \mathrm{mg} / \mathrm{dL}$ & $132 \pm 61$ & $118 \pm 41$ & $128 \pm 66$ & $150 \pm 68$ & 0.031 \\
\hline$<110, \%$ & 49.4 & 58.5 & 52.1 & 36.5 & 0.055 \\
\hline $110-126, \%$ & 11.9 & 15.1 & 12.7 & 7.7 & 0.368 \\
\hline $127-179, \%$ & 25.6 & 17.0 & 26.8 & 32.7 & 0.155 \\
\hline$\geq 180, \%$ & 13.1 & 9.4 & 8.5 & 23.1 & 0.154 \\
\hline
\end{tabular}

$\mathrm{SBP}=$ systolic blood pressure; $\mathrm{DBP}=$ diastolic blood pressure; $\mathrm{BMI}=$ body mass index $\mathrm{cr}=$ creatinine; $\mathrm{Alb}=\mathrm{albu}-$ $\min ;[x]_{p}=$ plasma concentration of $x ;[x]_{b}=$ blood concentration of $x ;[x]_{u}=$ urine concentration of $x ;$ eGFR $=$ estimated glomerular filtration rate; $\mathrm{ACR}=$ albumin-to-creatinine ratio; $[\mathrm{Glc}]_{\mathrm{p}}=$ fasting plasma glucose concentration a Profile 1 was defined as $[\mathrm{Cd}]_{\mathrm{b}}$ and $[\mathrm{Pb}]_{\mathrm{b}} \leq$ its respective median of $0.3 \mu \mathrm{g} / \mathrm{L}$ and $2.12 \mu \mathrm{g} / \mathrm{dL}$. Profile 2 was defined as $[\mathrm{Cd}]_{\mathrm{b}}$ or $[\mathrm{Pb}]_{\mathrm{b}} \geq$ its respective median. Profile 3 was defined as $[\mathrm{Cd}]_{b}$ and $[\mathrm{Pb}]_{b}>$ its respective median. ${ }^{b}$ Diabetes diagnosis was based on medical records. ${ }^{c}$ Hypertension is defined as SBP $\geq 140$ or DBP $\geq 90 \mathrm{mmHg}$. d BMI classification as thin, normal, overweight, and obesity correspond to BMI $<18,19-23,24-30$, and $>30 \mathrm{~kg} / \mathrm{m}^{2}$, respectively. ${ }^{\mathrm{e}}$ eGFR is determined with Chronic Kidney Disease Epidemiology Collaboration (CKD-EPI) equations. ${ }^{\mathrm{f}}[\mathrm{Glc}]_{\mathrm{p}} \geq 110,126$, and $180 \mathrm{mg} / \mathrm{dL}$ indicate impaired fasting plasma glucose, diabetes diagnosis, and the renal threshold for glucose. Data for all continuous variables are arithmetic means \pm standard deviation (SD) $p \leq 0.05$ identifies statistical significance, determined with the Pearson Chi-Square test for $\%$ differences, and the Kruskal-Wallis test for mean differences.

About half (52\%) were hypertensive, and 9.7\% were smokers. Over half (54\%) were overweight and $10.8 \%$ were obese. The overall mean for age was 59.9 years, and mean body mass index (BMI) was $25.4 \mathrm{~kg} / \mathrm{m}^{2}$. The overall mean blood Cd concentration $\left([\mathrm{Cd}]_{\mathrm{b}}\right)$ was $0.59 \mu \mathrm{g} / \mathrm{L}$, and the mean blood $\mathrm{Pb}$ concentration $\left([\mathrm{Pb}]_{\mathrm{b}}\right)$ was $4.67 \mu \mathrm{g} / \mathrm{dL}$ with the median $[\mathrm{Cd}]_{\mathrm{b}}$ as $0.3 \mu \mathrm{g} / \mathrm{L}$ and the median $[\mathrm{Pb}]_{\mathrm{b}}$ as $2.12 \mu \mathrm{g} / \mathrm{dL}$. The prevalence rate of eGFR) $\leq 60 \mathrm{~mL} / \mathrm{min} / 1.7 \mathrm{~m}^{2}$ (low eGFR) was $16.5 \%$, while ACR of 30-299 and $\geq 300 \mathrm{mg} / \mathrm{g}$ creatinine were $17.4 \%$ and $4.2 \%$, respectively. About half had fasting plasma glucose [Glc $]_{p}$ 
within a normal range $(<110 \mathrm{mg} / \mathrm{dL})$, while $11.9 \%, 25.6 \%$, and $13 \%$ of participants had $[\mathrm{Glc}]_{\mathrm{p}}$ of $110-125,126-179$, and $\geq 180 \mathrm{mg} / \mathrm{dL}$, respectively.

The $\%$ of participants with diabetes diagnosis was the highest $(69.2 \%)$, middle $(46.5 \%)$, and lowest $(35.8 \%)$ in profiles 3,2 , and $1(p=0.028)$. Likewise, smoking was the highest $(17.9 \%)$, middle $(9.9 \%)$, and lowest $(1.9 \%)$ in profiles 3,2 , and $1(p=0.028)$. The $\%$ of men and women across three exposure profiles was similar $(p=0.092)$ as was the $\%$ of hypertension $(p=0.067)$. The percentages of four BMI classes (thin, normal, overweight, and obesity) across three $\mathrm{Cd}$ and $\mathrm{Pb}$ exposure profiles did not differ.

The mean [Glc] was highest $(150 \mathrm{mg} / \mathrm{dL})$, middle $(128 \mathrm{mg} / \mathrm{dL})$, and lowest $(118 \mathrm{mg} / \mathrm{dL})$ in exposure profiles 3,2 , and $1(p=0.031)$. The means for age $(p=0.639)$, BMI $(p=0.426)$, and duration of diabetes $(p=0.910)$ did not differ nor did the means for [cr $]_{\mathrm{p}}(p=0.133)$, eGFR $(p=0.269), \operatorname{ACR}(p=0.389),[\mathrm{Alb}]_{\mathrm{u}}(p=0.564)$, and $[\mathrm{cr}]_{\mathbf{u}}(p=0.499)$. Likewise, the $\%$ of low eGFR, ACR of $30-299 \mathrm{mg} / \mathrm{g}$ creatinine, and $\geq 300 \mathrm{mg} / \mathrm{g}$ creatinine among groups did not differ $(p=0.169-0.786)$ nor did the $\%$ distribution of four [Glc $]_{p}$ levels $(p=0.154-0.368)$.

\subsection{Logistic Regression Analysis}

Results in Table 2 show that the POR for diabetes diagnosis did not associate with age, BMI, smoking status, hypertension, or gender $(p=0.115-0.457)$, but it increased by 3 -fold in participants with profile 2 (POR 2.991, 95\% CI: 1.317, 6.794, $p=0.009$ ), and by 4 -fold in those with profile 3 (POR 4.182, 95\% CI: 1.725, 10.14, $p=0.002$ ). The POR for diabetes diagnosis increased by 3 -fold in those with eGFR $\leq 60 \mathrm{~mL} / \mathrm{min} / 1.73 \mathrm{~m}^{2}$ (POR 2.926, 95\% CI: $1.115,7.679, p=0.029$ ).

Table 2. Logistic regression analysis of type 2 diabetes diagnosis.

\begin{tabular}{|c|c|c|c|c|c|c|}
\hline \multirow{3}{*}{$\begin{array}{c}\text { Independent } \\
\text { Variables/Factors }\end{array}$} & \multirow{3}{*}{$\begin{array}{l}\text { Number of } \\
\text { Subjects }\end{array}$} & \multicolumn{5}{|c|}{ Diabetes Diagnosis $^{a}$} \\
\hline & & \multirow{2}{*}{$\begin{array}{c}\beta \text { Coefficients } \\
\text { (SE) }\end{array}$} & \multirow[t]{2}{*}{ POR } & \multicolumn{2}{|c|}{$95 \%$ CI } & \multirow{2}{*}{$p$} \\
\hline & & & & Lower & Upper & \\
\hline Age, years & 173 & $0.014(0.019)$ & 1.015 & 0.977 & 1.054 & 0.457 \\
\hline $\mathrm{BMI}, \mathrm{kg} / \mathrm{m}^{2}$ & 173 & $-0.062(0.040)$ & 0.939 & 0.869 & 1.015 & 0.115 \\
\hline Smoking status & 173 & $-0.951(0.723)$ & 0.386 & 0.094 & 1.593 & 0.188 \\
\hline Hypertension & 173 & $-0.321(0.334)$ & 0.725 & 0.377 & 1.394 & 0.335 \\
\hline Gender & 173 & $-0.450(0.542)$ & 0.638 & 0.220 & 1.846 & 0.407 \\
\hline \multicolumn{7}{|l|}{$\mathrm{eGFR}, \mathrm{mL} / \mathrm{min} / 1.73 \mathrm{~m}^{2}$} \\
\hline$>60$ & 144 & Referent & & & & \\
\hline$\leq 60$ & 29 & $1.074(0.492)$ & 2.926 & 1.115 & 7.679 & 0.029 \\
\hline \multicolumn{7}{|l|}{$\mathrm{Cd}-\mathrm{Pb}$ exposure ${ }^{b}$} \\
\hline Profile $1^{\mathrm{c}}$ & 50 & Referent & & & & \\
\hline Profile 2 & 71 & $1.096(0.419)$ & 2.991 & 1.317 & 6.794 & 0.009 \\
\hline Profile 3 & 52 & $1.431(0.452)$ & 4.182 & 1.725 & 10.14 & 0.002 \\
\hline
\end{tabular}

$\mathrm{POR}=$ prevalence odds ratio; $\mathrm{CI}=$ confidence interval; $\mathrm{BMI}=$ body mass index; $[\mathrm{Glc}]_{\mathrm{p}}=$ fasting plasma glucose level. Coding; non-smoker $=1$; smokers $=2$; hypertensive $=1$; normotensive $=2$; male $=1$; female $=2$. ${ }^{\text {a }} \mathrm{A}$ diagnosis of type 2 diabetes was based on a medical record of each subject. ${ }^{\mathrm{b}}$ Profile 1 was defined as $[\mathrm{Cd}]_{\mathrm{b}}$ and $[\mathrm{Pb}]_{\mathrm{b}} \leq$ its respective median of $0.3 \mu \mathrm{g} / \mathrm{L}$ and $2.12 \mathrm{mg} / \mathrm{dL}$. Profile 2 was defined as $[\mathrm{Cd}]_{\mathrm{b}}$ or $[\mathrm{Pb}]_{\mathrm{b}} \geq$ its respective median. Profile 3 was defined as $[\mathrm{Cd}]_{\mathrm{b}}$ and $[\mathrm{Pb}]_{\mathrm{b}}>$ its respective median. ${ }^{\mathrm{c}}$ Blood pressure data were missing in three subjects.

Results in Table 3 indicate that POR for [Glc] p equal to or above the renal threshold for glucose of $180 \mathrm{mg} / \mathrm{dL}$ increased slightly with age (POR 1.066, 95\% CI: 1.011,1.124, $p=0.018)$, while POR for [Glc $]_{\mathrm{p}} \geq 110 \mathrm{mg} / \mathrm{dL}(p=0.653)$ and for $[\mathrm{Glc}]_{\mathrm{p}} \geq 126 \mathrm{mg} / \mathrm{dL}$ $(p=0.073)$ did not associate with age. POR values for all three levels of abnormal [Glc $]_{p}$ did not associate with BMI ( $p=0.095)$ or smoking $(p=-0.301)$. In the profile 2 group, an increment of POR for $[\mathrm{Glc}]_{\mathrm{p}} \geq 110 \mathrm{mg} / \mathrm{dL}$ did not reach statistical significance $(p=0.086)$. However, in this profile 2 group, the POR [Glc $]_{p} \geq 126$ and $\geq 180 \mathrm{mg} / \mathrm{dL}$ rose, respectively, by 2.29 - (95\% POR 2.290, CI: 1.073, 4.885, $p=0.032$ ) and 3.38-fold (95\% CI: $1.136,10.08$, $p=0.029$ ). In the profile 3 group, the POR for [Glc $]_{\mathrm{p}} \geq 110, \geq 126$, and $\geq 180 \mathrm{mg} / \mathrm{dL}$ rose 
by 2.79 - (95\% CI: 1.228, 6.375, $p=0.014), 2.964-(95 \%$ CI:1.288, 6.822, $p=0.011)$, and 3.41-fold (95\% CI: $1.061,10.94, p=0.039)$, respectively.

Table 3. Logistic regression modeling of abnormally high fasting plasma glucose levels.

\begin{tabular}{|c|c|c|c|c|c|c|}
\hline \multirow{2}{*}{$\begin{array}{l}\text { Independent } \\
\text { Variables/Factors }\end{array}$} & \multicolumn{2}{|c|}{ Model 1 ${ }^{\mathrm{a}}, n 173$} & \multicolumn{2}{|c|}{ Model $2^{b}, n 173$} & \multicolumn{2}{|c|}{ Model $3^{c}, n 173$} \\
\hline & POR (95\% CI) & $p$ & POR (95\% CI) & $p$ & POR (95\% CI) & $p$ \\
\hline Age, years & $1.008(0.975,1.042)$ & 0.653 & $1.033(0.997,1.069)$ & 0.073 & $1.066(1.011,1.124)$ & 0.018 \\
\hline BMI, $\mathrm{kg} / \mathrm{m}^{2}$ & $0.940(0.875,1.011)$ & 0.095 & $0.958(0.891,1.029)$ & 0.238 & $1.062(0.959,1.176)$ & 0.250 \\
\hline Smoking status & $0.567(0.194,1.662)$ & 0.301 & $0.638(0.209,1.941)$ & 0.428 & $0.246(0.029,2.063)$ & 0.196 \\
\hline \multicolumn{7}{|l|}{$\mathrm{Cd}-\mathrm{Pb}$ exposure $\mathrm{d}$} \\
\hline Profile 1 & Referent & & Referent & & Referent & \\
\hline Profile 2 & $1.940(0.911,4.132)$ & 0.086 & $2.290(1.073,4.885)$ & 0.032 & $3.383(1.136,10.08)$ & 0.029 \\
\hline Profile 3 & $2.794(1.228,6.375)$ & 0.014 & $2.964(1.288,6.822)$ & 0.011 & $3.407(1.061,10.94)$ & 0.039 \\
\hline
\end{tabular}

$[\mathrm{Glc}]_{\mathrm{p}}=$ fasting plasma glucose level; POR = prevalence odds ratio; BMI = body mass index. Coding; nonsmoker $=1$; smokers $=2$. ${ }^{\text {a }}$ Model 1 outcome $=[\mathrm{Glc}]_{\mathrm{p}} \geq 110 \mathrm{mg} / \mathrm{dL} .{ }^{\mathrm{b}}$ Model 2 outcome $=[\mathrm{Glc}]_{\mathrm{p}} \geq 126 \mathrm{mg} / \mathrm{dL}$. ${ }^{\mathrm{c}}$ Model 3 outcome $=[\mathrm{Glc}]_{\mathrm{p}} \geq 180 \mathrm{mg} / \mathrm{dL}$. ${ }^{\mathrm{d}}$ Profile 1 was defined as $[\mathrm{Cd}]_{\mathrm{b}}$ and $[\mathrm{Pb}]_{\mathrm{b}} \leq$ its respective median. Profile 2 was defined as $[\mathrm{Cd}]_{\mathrm{b}}$ or $[\mathrm{Pb}]_{\mathrm{b}} \geq$ its respective median. Profile 3 was defined as $[\mathrm{Cd}]_{\mathrm{b}}$ and $[\mathrm{Pb}]_{\mathrm{b}}>$ its respective median.

\subsection{Correlation Analysis}

Of eight variables tested, $[\mathrm{Glc}]_{\mathrm{p}}$ correlated significantly with four variables, including age, $[\mathrm{Pb}]_{b}, \mathrm{ACR}$, and exposure profiles (Table 4). An inverse correlation was seen between $[\mathrm{Glc}]_{\mathrm{p}}$ and age $(r=-0.193)$, while positive correlations were seen between $[\mathrm{Glc}]_{\mathrm{p}}$ and $[\mathrm{Pb}]_{\mathrm{b}}$ $(r=0.194)$, ACR $(r=0.288)$, and exposure profiles $(r=0.216)$.

Table 4. Bivariate correlation analysis of fasting plasma glucose levels.

\begin{tabular}{|c|c|c|c|c|c|c|c|c|c|}
\hline \multirow{2}{*}{ Variables } & \multicolumn{9}{|c|}{ Pearson's Correlation Coefficients } \\
\hline & {$[\mathrm{Glc}]_{\mathrm{b}}$} & Age & BMI & Gender & SMK & {$[\mathrm{Pb}]_{\mathbf{b}}$} & {$[\mathrm{Cd}]_{\mathrm{b}}$} & ACR & eGFR \\
\hline$[\mathrm{Glc}]_{\mathrm{b}}$ & 1 & & & & & & & & \\
\hline Age & $-0.193 *$ & 1 & & & & & & & \\
\hline BMI & 0.078 & $-0.285^{* * *}$ & 1 & & & & & & \\
\hline Gender & -0.057 & $0.186^{*}$ & 0.140 \# & 1 & & & & & \\
\hline SMK & -0.040 & -0.018 & $-0.152 *$ & $-0.620^{* * *}$ & 1 & & & & \\
\hline$[\mathrm{Pb}]_{\mathrm{b}}$ & 0.194 * & -0.071 & 0.060 & -0.108 & 0.153 * & 1 & & & \\
\hline$[\mathrm{Cd}]_{\mathrm{b}}$ & 0.049 & 0.053 & -0.047 & -0.034 & 0.184 * & $0.148 *$ & 1 & & \\
\hline ACR & $0.288^{* * *}$ & 0.114 & 0.003 & 0.009 & -0.031 & 0.047 & 0.098 & 1 & \\
\hline eGFR & 0.092 & $-0.454^{* * *}$ & $0.172 *$ & -0.139 & 0.040 & 0.090 & -0.123 & $-0.176^{*}$ & 1 \\
\hline $\mathrm{Cd} / \mathrm{Pb}$ profiles & $0.216^{* *}$ & -0.022 & -0.017 & -0.134 & $0.202 * *$ & $0.604^{* *}$ & $0.724^{* * *}$ & 0.102 & -0.008 \\
\hline
\end{tabular}

$[\mathrm{Glc}]_{\mathrm{b}}$ fasting blood glucose levels as $\log [\mathrm{Glc}]_{\mathrm{b}} ; \mathrm{BMI}=$ body mass index; $\mathrm{SMK}=$ smoking status; $[\mathrm{Pb}]_{\mathrm{b}}=$ blood concentration of $\mathrm{Pb}$ as $\log \left([\mathrm{Pb}]_{\mathrm{b}}\right) \times 100 ;[\mathrm{Cd}]_{\mathrm{b}}=$ blood concentration of $\mathrm{Cd}$ as $\log \left([\mathrm{Cd}]_{\mathrm{b}}\right) \times 100 ; \mathrm{ACR}$ as $\log \left[(\mathrm{ACR}) \times 10^{3}\right]$. Coding: male $=1$; female $=2$; nonsmoker $=1$; smoker $=2$; profile $1=1$; profile $2=2$; profile $3=3$. $p$-values $\leq 0.05$ indicate statistically significant correlation of individual pairs of variables. $\# p=0.054$; ${ }^{*} p=0.010-0.050 ;{ }^{* *} p=0.004-0.007 ;{ }^{* * *} p<0.001$.

\subsection{Covariance Analysis of Fasting Plasma Glucose Variation}

Figure 1 depicts results of an analysis of the variation in $[\mathrm{Glc}]_{\mathrm{p}}$ across three exposure groups, and subgroups, stratified by exposure profiles and blood pressure status.

Because the prevalence of smoking among participants was low $(9.7 \%)$ and there was a gender bias (of 18 smokers, one was woman), the mean for fasting plasma glucose derived for each $\mathrm{Cd}-\mathrm{Pb}$ exposure profile was not adjusted for smoking. The means for fasting plasma glucose were adjusted for age and BMI. Because $\mathrm{Cd}$ and $\mathrm{Pb}$ both are cumulative toxicants, the body burden of these metals increases with age. Obesity $\left(\mathrm{BMI}>30 \mathrm{~kg} / \mathrm{m}^{2}\right)$ is a known risk factor of diabetes and hypertension. $\mathrm{Cd}$ and $\mathrm{Pb}$ exposure profiles marginally accounted for the variation in $[\mathrm{Glc}]_{\mathrm{p}}\left(F=2.781, \eta^{2}=0.033, p=0.065\right)$ (Figure 1a). A subgroup analysis showed that $[\mathrm{Glc}]_{\mathrm{p}}$ was elevated, especially in participants with profile 3 who also 
had hypertension $(p=0.033)$, compared to those of the same exposure profile whose blood pressure was within a normal range (Figure 1b).

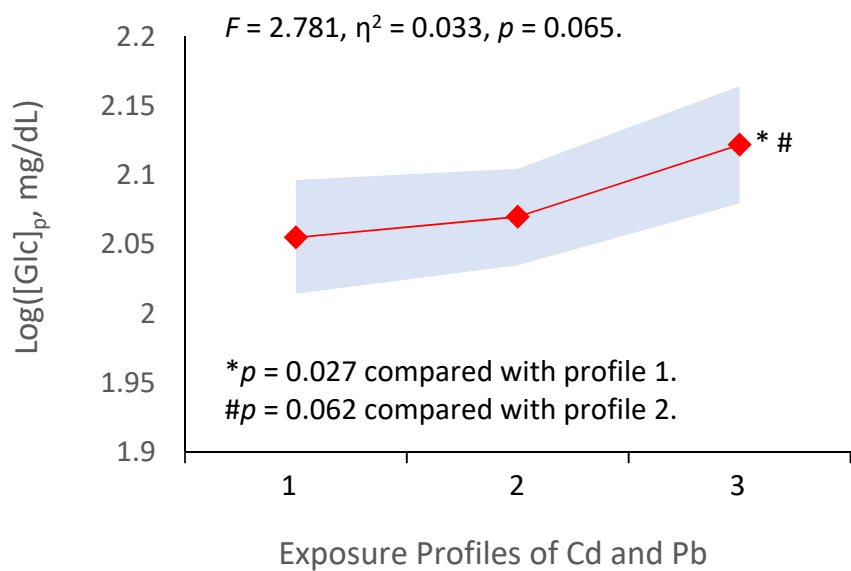

(a)

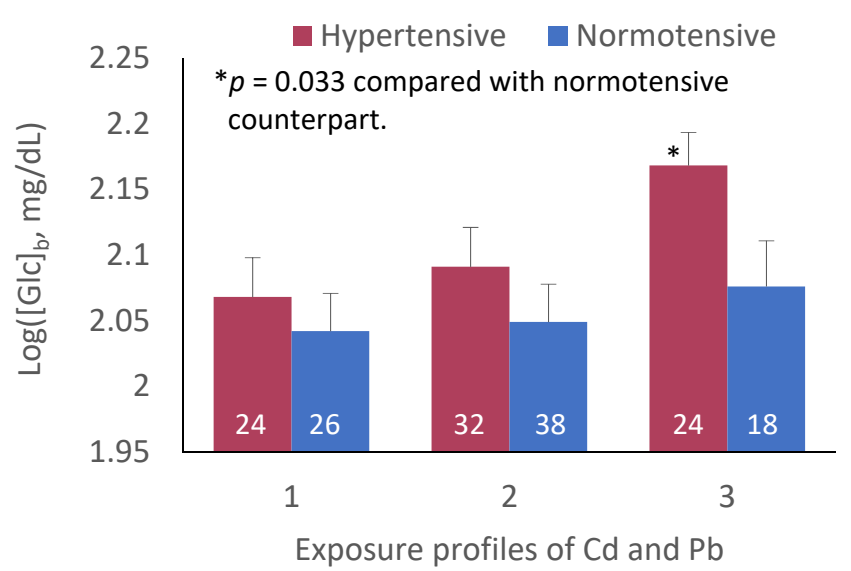

(b)

Figure 1. Association of cadmium and lead exposure profiles with fasting plasma glucose variation. The color-coded area graph depicts means for fasting plasma glucose levels as $\log [\mathrm{Glc}]_{\mathrm{p}}$ in participants with exposure profiles 1, 2, and 3 together with variability of the means (a). The bar graphs compare means for fasting plasma glucose as $\log [G l c]_{p}$ in participants with and without hypertension across three profiles (b). The number of participants with profiles 1, 2, and 3 are 50, 70, and 52, respectively. The means are obtained by univariate/covariance analysis, adjusted for age and BMI. Bonferroni correction is applied to multiple comparisons.

Table 5 shows results of logistic regression analysis of albuminuria in relation to low eGFR and abnormal fasting [Glc]p levels.

Table 5. Association of the prevalence odds for albuminuria with abnormal fasting plasma glucose levels.

\begin{tabular}{|c|c|c|c|c|c|c|}
\hline \multirow{3}{*}{$\begin{array}{c}\text { Independent } \\
\text { Variables/Factors }\end{array}$} & \multirow{3}{*}{$\begin{array}{l}\text { Number of } \\
\text { Subjects } b\end{array}$} & \multicolumn{5}{|c|}{ Albuminuria $^{a}$} \\
\hline & & \multirow{2}{*}{$\begin{array}{c}\beta \text { Coefficients } \\
\text { (SE) }\end{array}$} & \multirow[t]{2}{*}{ POR } & \multicolumn{2}{|c|}{$95 \%$ CI } & \multirow{2}{*}{$p$} \\
\hline & & & & Lower & Upper & \\
\hline Age, years & 164 & $-0.040(0.028)$ & 0.961 & 0.910 & 1.014 & 0.147 \\
\hline BMI, $\mathrm{kg} / \mathrm{m}^{2}$ & 164 & $-0.027(0.047)$ & 0.974 & 0.888 & 1.068 & 0.570 \\
\hline Smoking status & 164 & $-1.287(0.914)$ & 0.276 & 0.046 & 1.656 & 0.159 \\
\hline Hypertension & 164 & $-1.225(0.482)$ & 0.294 & 0.114 & 0.755 & 0.011 \\
\hline Gender & 164 & $-1.825(0.644)$ & 0.161 & 0.046 & 0.570 & 0.005 \\
\hline \multicolumn{7}{|l|}{$\mathrm{eGFR}, \mathrm{mL} / \mathrm{min} / 1.73 \mathrm{~m}^{2}$} \\
\hline$>60$ & 138 & Referent & & & & \\
\hline$\leq 60$ & 26 & $1.129(0.575)$ & 3.093 & 1.002 & 9.552 & 0.050 \\
\hline \multicolumn{7}{|l|}{$[\mathrm{Glc}]_{\mathrm{p}}, \mathrm{mg} / \mathrm{dL}$} \\
\hline$<110$ & 81 & Referent & & & & \\
\hline $110-126$ & 20 & $0.453(0.634)$ & 1.574 & 0.454 & 5.457 & 0.475 \\
\hline $127-179$ & 43 & $1.210(0.816)$ & 3.353 & 0.678 & 16.58 & 0.138 \\
\hline$\geq 180$ & 20 & $1.597(0.653)$ & 4.937 & 1.373 & 17.76 & 0.014 \\
\hline
\end{tabular}

${ }^{a}$ Albuminuria is defined as albumin to creatinine ratio $\geq 20 \mathrm{mg} / \mathrm{g}$ for men, and $\geq 30 \mathrm{mg} / \mathrm{g}$ for women. ${ }^{\mathrm{b}}$ Urine albumin or blood pressure data were missing in 12 participants. $\mathrm{POR}=$ prevalence odds ratio; $\mathrm{CI}=$ confidence interval; $\mathrm{BMI}=$ body mass index; $[\mathrm{Glc}]_{\mathrm{p}}=$ fasting plasma glucose level. Coding; non-smoker $=1$; smokers $=2$; hypertensive $=1 ;$ normotensive $=2 ;$ male $=1$; female $=2$.

By logistic regression analysis (Table 5), the POR for albuminuria was increased by 5-fold in participants with [Glc] $\mathrm{p} \geq 180 \mathrm{mg} / \mathrm{dL}$ (POR 4.937, 95\% CI: 1.373, 17.76, $p=0.014$ ), 
compared with those with [Glc] $]_{p}<110 \mathrm{mg} / \mathrm{dL}$. It rose by 3-fold in those with low eGFR (POR 3.093, 95\% CI: 1.002, 9.552, $p=0.050$ ). POR for albuminuria fell by $70 \%$ in the normotensive relative to the hypertensive (POR 0.294, 95\% CI: $0.114,0.755, p=0.011$ ). It also fell by $84 \%$ in women compared with men (POR $=0.161,95 \%$ CI: $0.046,0.570, p=0.005)$.

\section{Discussion}

In the present study, we examined the effects of simultaneous exposure to $\mathrm{Cd}$ and $\mathrm{Pb}$ on fasting plasma glucose levels, and clinical kidney functional measures, low eGFR, and albuminuria. In total, $65.4 \%$ of the cohort had $[\mathrm{Cd}]_{\mathrm{b}}$ above the detection limit, while $46.5 \%$ had $[\mathrm{Pb}]_{\mathrm{b}}$ above the detection limit. This suggests that $\mathrm{Cd}$ exposure was more widespread than exposure to $\mathrm{Pb}$. There was a wider variation in $\mathrm{Cd}$ exposure levels than $\mathrm{Pb}$ among subjects. Those subjects in the profile 3 group, where the mean $[\mathrm{Cd}]_{\mathrm{b}}$ was $1.05 \mu \mathrm{g} / \mathrm{L}$, had 1.62- and 21-fold higher $[\mathrm{Cd}]_{\mathrm{b}}$ than those with profiles 2 and 1, respectively. The $[\mathrm{Cd}]_{\mathrm{b}}$ levels among those with profiles 2 and 3 were in those ranges found to be associated with increased risk of CKD in the representative U.S. population $[13,14]$. $[\mathrm{Cd}]_{\mathrm{b}}$ levels $\geq 0.61 \mu \mathrm{g} / \mathrm{L}$ in adult participants in NHANES 2007-2012 were associated with 1.8- and 2.2-fold increases in risk of low eGFR and albuminuria, respectively [13]. [Cd] $]_{b}$ levels $\geq 0.53 \mu \mathrm{g} / \mathrm{L}$ in adult participants in NHANES 2011-2012 were associated with 2.21and 2.04-fold increases in the risk of low eGFR and albuminuria, respectively [14].

Those subjects in the profile 3 group, where the mean $[\mathrm{Pb}]_{\mathrm{b}}$ was $7.38 \mu \mathrm{g} / \mathrm{dL}$, had 1.61and 3.48-fold higher $[\mathrm{Pb}]_{b}$ than those with profiles 2 and 1, respectively. Compared to $[\mathrm{Pb}]_{b}$ of $0.5 \mu \mathrm{g} / \mathrm{dL}$, the level that has not been found to be associated with an adverse effect in adults in any epidemiologic studies [39], the mean $[\mathrm{Pb}]_{\mathrm{b}}$ recorded for this study group of $4.67 \mu \mathrm{g} / \mathrm{dL}$ was 10 times higher. Indeed, $[\mathrm{Pb}]_{\mathrm{b}}$ levels $\geq 2.4 \mu \mathrm{g} / \mathrm{dL}$ in adult participants in NHANES 1999-2006 were associated with 1.56-fold increase in the risk of low eGFR [11]. $[\mathrm{Pb}]_{\mathrm{b}}$ levels $\geq 3.3 \mu \mathrm{g} / \mathrm{dL}$ were associated with 1.49-fold increase in the incidence of CKD, while $[\mathrm{Pb}]_{\mathrm{b}}$ of $7.6 \mu \mathrm{g} / \mathrm{dL}$ was associated with an increase in the risk of ESKD in prospective cohort studies of the Swedish population [21,22].

In the present study, BMI was not a significant predictor of diabetes diagnosis (Table 2), nor was it associated with abnormal fasting plasma glucose levels (Table 3). Similarly, obesity was not related to $\mathrm{Cd}$ and $\mathrm{Pb}$ exposure profile (Table 1). This observation was consistent with results of studies in the U.S. [40,41], Canada [42], Korea [5], and China [6] showing an inverse relationship between measures of obesity and $\mathrm{Cd}$ exposure estimates such as $[\mathrm{Cd}]_{\mathrm{b}},[\mathrm{Cd}]_{\mathrm{u}}$, or $\mathrm{E}_{\mathrm{Cd}}$. Among participants of NHANES 1999-2002, an inverse association between $[\mathrm{Cd}]_{\mathrm{u}}$ and central obesity was observed [40], while an inverse association between $[\mathrm{Cd}]_{b}$ and BMI was seen in the NHANES 2003-2010 cycle [41]. Similarly, an inverse association between $[\mathrm{Cd}]_{b}$ and BMI was seen in non-smokers in the Canadian Health Survey 2007-2011 [42]. A negative association between Cd exposure and various obesity measures was seen in both men and women in a study of the indigenous population of Northern Québec, Canada, where obesity was highly prevalent [43]. An inverse association between $[\mathrm{Cd}]_{b}$ and BMI was noted in a group of Korean men, 40-70 years of age, showing a mean for $[\mathrm{Cd}]_{\mathrm{b}}$ of $1.7 \mu \mathrm{g} / \mathrm{L}$, and a mean for $\mathrm{E}_{\mathrm{Cd}}$ of $2.13 \mu \mathrm{g} / \mathrm{g}$ creatinine [5], while an inverse association between $\mathrm{E}_{\mathrm{Cd}}$ levels $\geq 2.95 \mu \mathrm{g} / \mathrm{g}$ creatinine and reduced risk of overweight was reported by a Chinese study [6]. Urinary $\mathrm{Cd}$ levels were inversely associated with height and BMI in a study of Flemish children [44]. An association between urinary $\mathrm{Cd}$ and a reduction in risk of obesity by $54 \%$ was seen in 6-19 year old children and adolescents, enrolled in NHANES 1999-2011 [45].

The prevalence of abnormally high fasting $[\mathrm{Glc}]_{\mathrm{p}}$ in this study group was high, 50.6\%. Three subjects recruited as non-diabetic controls had [Glc]p levels higher than a diabetes diagnosis level. One of these three subjects had exposure profile 3, while the other two had exposure profile 1 . For the whole group, one in four $(25.6 \%)$ had [Glc]p between 127 and $179 \mathrm{mg} / \mathrm{dL}$, and $13.1 \%$ had $[\mathrm{Glc}]_{\mathrm{p}}$ exceeding the kidney threshold for glucose of $180 \mathrm{mg} / \mathrm{dL}$, and all these 23 subjects had $\mathrm{Cd}-\mathrm{Pb}$ exposure profile 3 . The severity of impaired glycemic control, and exposure outcome as albuminuria both were related to 
their exposure profiles and the presence of hypertension (Figure 1b). It should, however, be noted that hypertension can be a cause or a consequence of CKD since blood pressure rises when GFR falls. Exposure profile 3 was associated with a 3.4-fold increase in the POR for $[\mathrm{Glc}]_{\mathrm{p}} \geq 180 \mathrm{mg} / \mathrm{dL}(p=0.039)$ (Table 3). The POR for albuminuria increased by 5-fold in those with [Glc $]_{p} \geq 180 \mathrm{mg} / \mathrm{dL}$ (Table 5). Our findings underscore the conclusion from the nationwide Thai diabetes cohort study that glycemic control was more effective than other measures, such as blood pressure control, to delay the progression of kidney disease in patients with type 2 diabetes [46]. Notably, however, to achieve the target glycemic control, avoidance of high dietary intake of $\mathrm{Cd}$ and $\mathrm{Pb}$ may also be necessary.

It is noteworthy that the worldwide rising incidence of type 2 diabetes mellitus has often been linked to increasing prevalence of obesity, but studies in various populations, described above, found an inverse association between $\mathrm{Cd}$ exposure estimates and body weight gain and other measures of obesity. Consequently, exposure to $\mathrm{Cd}$, especially of dietary origin, appears to be a contributor to the global increase in prevalence of diabetes.

Herein, we provide, for the first time, data that link simultaneous exposure to $\mathrm{Cd}$ and $\mathrm{Pb}$ to increased risks of abnormally high fasting plasma glucose, and kidney dysfunction, evident from changes in GFR and albumin excretion rate. The reasons for the GFR reduction seen in people with diabetes have been not been investigated adequately although the potential involvement of environmental exposure to $\mathrm{Cd}$ and $\mathrm{Pb}$ has been suggested by crosssectional studies and longitudinal cohort studies [11-15,21,22]. We hypothesize that the low eGFR seen in those with high and moderate exposure to $\mathrm{Cd}$ and $\mathrm{Pb}$, was a consequence of $\mathrm{Cd}$ toxicity in proximal tubular cells, known to accumulate $\mathrm{Cd}[23,24,47]$. Abundant evidence suggests that $\mathrm{Cd}$ inflicts tubular cell injury at a low intracellular concentration of $\mathrm{Cd}$ and intensifies as the concentration rises. Inflammation and fibrosis follow, nephrons are lost, and GFR falls [23,24,47-50]. After substantial proximal tubular injury has occurred, reabsorption of albumin decreases and its excretion exceeds the normal limit.

The majority of epidemiological studies have considered $\mathrm{Cd}$ or $\mathrm{Pb}$ exposure, independently. Hence, our knowledge of the combined nephrotoxicity of low environmental exposure to $\mathrm{Cd}$ plus $\mathrm{Pb}$ has been limited. In the joint effect analysis using NHANES 1999-2006 data [13], [Cd] $]_{b}$ levels $\geq 0.6 \mu \mathrm{g} / \mathrm{L}$ were found to be associated with increases in risk of low eGFR and albuminuria by 1.53- and 1.92-fold, respectively. The increases in risk for low eGFR and albuminuria rose, respectively, to 1.98, and 2.34 in those who had $[\mathrm{Cd}]_{\mathrm{b}} \geq 0.6 \mu \mathrm{g} / \mathrm{L}$ plus $[\mathrm{Pb}]_{\mathrm{b}} \geq 2.4 \mu \mathrm{g} / \mathrm{dL}$.

Of note, a dose-response meta-analysis has shown that the risk of diabetes increases, when $[\mathrm{Cd}]_{\mathrm{b}}$ levels rise to levels above $1 \mu \mathrm{g} / \mathrm{L}$ [51]. However, data from the present study, and various NHANES cycles, discussed above, have linked $[\mathrm{Cd}]_{\mathrm{b}}$ levels of $0.5-0.6 \mu \mathrm{g} / \mathrm{L}$ to increases in the risk of GFR reduction and albuminuria. Thus, $\mathrm{Cd}$-induced nephropathy may contribute, in part, to an elevation of plasma glucose levels among the subjects in the present study, which accentuates the central role of kidneys in the maintenance of plasma glucose levels $[52,53]$. These notions are in line with experimental studies in rats, where daily subcutaneous doses of Cd at $0.6 \mathrm{mg} / \mathrm{kg}$ for 12 weeks caused proteinuria [54], and hyperglycemia [55]. Half of the subjects in the present study were diagnosed with type 2 diabetes (Table 2), and those with $\mathrm{Cd}$ and $\mathrm{Pb}$ exposure profiles 2 and 3 faced a $3-4$.2-fold increment in the likelihood of having a diabetes diagnosis $(p=0.002-0.009)$, while subjects with low eGFR were 2.9-times more likely to be diagnosed with diabetes. These findings call for public measures to reduce environmental pollution by $\mathrm{Cd}$ and $\mathrm{Pb}$ and their food-chain transference to minimize $\mathrm{Cd}$ - and $\mathrm{Pb}$-induced nephropathy.

\section{Conclusions}

We observed for the first time that people chronically exposed to $\mathrm{Cd}$ and $\mathrm{Pb}$ have enhanced risks of hyperglycemia, GFR reduction, and albuminuria. It is likely that one or both metals cause these adverse outcomes. The levels of exposure to environmental $\mathrm{Cd}$ and $\mathrm{Pb}$ among study subjects could be considered to be low to moderate, reflected by the arithmetic means (SD) for $[\mathrm{Cd}]_{\mathrm{b}}$ and $[\mathrm{Pb}]_{\mathrm{b}}$ of $0.59(0.74) \mu \mathrm{g} / \mathrm{L}$ and $4.67(4.88) \mu \mathrm{g} / \mathrm{dL}$, 
respectively. However, the evidence linking these levels of environmental exposure to $\mathrm{Cd}$ and $\mathrm{Pb}$ to impaired glycemic control, reflected by abnormally high fasting plasma glucose levels, has emerged. Furthermore, plasma glucose levels above the kidney threshold for glucose of $180 \mathrm{mg} / \mathrm{dL}$ was associated with 5-fold increase in the POR for albuminuria. Research to elucidate the mechanism(s) underlying the association of abnormally high plasma glucose and exposure to $\mathrm{Cd}$ and $\mathrm{Pb}$ is warranted.

Author Contributions: Conceptualization, S.Y. and S.S.; methodology, S.Y., P.P. and T.K., formal analysis, S.Y., P.P. and S.S.; investigation, S.Y., P.P., T.K., S.K. and P.W.; resources, S.Y., D.A.V. and G.C.G.; original draft preparation, S.Y. and S.S.; review and editing, D.A.V. and G.C.G. All authors have read and agreed to the published version of the manuscript.

Funding: This research was funded by the Research Grant, WU-IRG-63-026, Walailak University, Nakhon Si Thammarat Province, Thailand.

Institutional Review Board Statement: This study was conducted in accordance with the guidelines of the Declaration of Helsinki and approved by the Office of the Human Research Ethics Committee of Walailak University, Nakhon Si Thammarat Province, Thailand. Approval number WUEC-20-132-01, 28 May 2020.

Informed Consent Statement: All participants provided written informed consent.

Data Availability Statement: All data are contained within this article.

Acknowledgments: We thank the staff of Pakpoon Health Promoting Hospital, Pakpoon Municipality, Nakhon Si Thammarat Province, Thailand for their assistance with data collection. We thank Kenneth R. Phelps for his insightful comments and clinical interpretation guidance.

Conflicts of Interest: The authors have declared no potential conflict of interest.

\section{References}

1. Schwartz, G.G.; Il'yasova, D.; Ivanova, A. Urinary cadmium, impaired fasting glucose, and diabetes in the NHANES III. Diabetes Care 2003, 26, 468-470. [CrossRef] [PubMed]

2. Wallia, A.; Allen, N.B.; Badon, S.; El Muayed, M. Association between urinary cadmium levels and prediabetes in the NHANES 2005-2010 population. Int. J. Hyg. Environ. Health 2014, 217, 854-860. [CrossRef] [PubMed]

3. Little, B.B.; Reilly, R.; Walsh, B.; Vu, G.T. Cadmium is associated with type 2 diabetes in a Superfund Site Lead Smelter community in Dallas, Texas. Int. J. Environ. Res. Public Health 2020, 17, 4558. [CrossRef]

4. Shi, P.; Yan, H.; Fan, X.; Xi, S. A benchmark dose analysis for urinary cadmium and type 2 diabetes mellitus. Environ. Pollut. 2021, 273, 116519. [CrossRef]

5. Son, H.S.; Kim, S.G.; Suh, B.S.; Park, D.U.; Kim, D.S.; Yu, S.D.; Hong, Y.S.; Park, J.D.; Lee, B.K.; Moon, J.D.; et al. Association of cadmium with diabetes in middle-aged residents of abandoned metal mines: The first health effect surveillance for residents in abandoned metal mines. Ann. Occup. Environ. Med. 2015, 27, 20. [CrossRef]

6. Nie, X.; Wang, N.; Chen, Y.; Chen, C.; Han, B.; Zhu, C.; Chen, Y.; Xia, F.; Cang, Z.; Lu, M.; et al. Blood cadmium in Chinese adults and its relationships with diabetes and obesity. Environ. Sci. Pollut. Res. Int. 2016, 23, 18714-18723. [CrossRef] [PubMed]

7. Simić, A.; Hansen, A.F.; Åsvold, B.O.; Romundstad, P.R.; Midthjell, K.; Syversen, T.; Flaten, T.P. Trace element status in patients with type 2 diabetes in Norway: The HUNT3 Survey. J. Trace Elem. Med. Biol. 2017, 41, 91-98. [CrossRef]

8. Hansen, A.F.; Simić, A.; Åsvold, B.O.; Romundstad, P.R.; Midthjell, K.; Syversen, T.; Flaten, T.P. Trace elements in early phase type 2 diabetes mellitus-A population-based study. The HUNT study in Norway. J. Trace Elem. Med. Biol. 2017, 40, 46-53. [CrossRef]

9. Grau-Perez, M.; Pichler, G.; Galan-Chilet, I.; Briongos-Figuero, L.S.; Rentero-Garrido, P.; Lopez-Izquierdo, R.; Navas-Acien, A.; Weaver, V.; García-Barrera, T.; Gomez-Ariza, J.L.; et al. Urine cadmium levels and albuminuria in a general population from Spain: A gene-environment interaction analysis. Environ. Int. 2017, 106, 27-36. [CrossRef]

10. Myong, J.P.; Kim, H.R.; Baker, D.; Choi, B. Blood cadmium and moderate-to-severe glomerular dysfunction in Korean adults: Analysis of KNHANES 2005-2008 data. Int. Arch. Occup. Environ. Health 2012, 85, 885-893. [CrossRef]

11. Navas-Acien, A.; Tellez-Plaza, M.; Guallar, E.; Muntner, P.; Silbergeld, E.; Jaar, B.; Weaver, V. Blood cadmium and lead and chronic kidney disease in US adults: A joint analysis. Am. J. Epidemiol. 2009, 170, 1156-1164. [CrossRef] [PubMed]

12. Ferraro, P.M.; Costanzi, S.; Naticchia, A.; Sturniolo, A.; Gambaro, G. Low level exposure to cadmium increases the risk of chronic kidney disease: Analysis of the NHANES 1999-2006. BMC Public Health 2010, 10, 304. [CrossRef] [PubMed]

13. Madrigal, J.M.; Ricardo, A.C.; Persky, V.; Turyk, M. Associations between blood cadmium concentration and kidney function in the U.S. population: Impact of sex, diabetes and hypertension. Environ. Res. 2018, 169, 180-188. [CrossRef] [PubMed]

14. Lin, Y.S.; Ho, W.C.; Caffrey, J.L.; Sonawane, B. Low serum zinc is associated with elevated risk of cadmium nephrotoxicity. Environ. Res. 2014, 134, 33-38. [CrossRef] [PubMed] 
15. Lee, J.; Oh, S.; Kang, H.; Kim, S.; Lee, G.; Li, L.; Kim, C.T.; An, J.N.; Oh, Y.K.; Lim, C.S.; et al. Environment-wide association study of CKD. Clin. J. Am. Soc. Nephrol. 2020, 15, 766-775. [CrossRef] [PubMed]

16. Soveri, I.; Berg, U.B.; Bjork, J.; Elinder, C.-G.; Grubb, A.; Mejare, I.; Sterner, G.; Sten-Erik Bäck, S.-E.; SBU GFR Review Group. Measuring GFR: A systematic review. Am. J. Kidney Dis. 2014, 64, 411-424. [CrossRef]

17. Levey, A.S.; Becker, C.; Inker, L.A. Glomerular filtration rate and albuminuria for detection and staging of acute and chronic kidney disease in adults: A systematic review. JAMA 2015, 313, 837-846. [CrossRef]

18. White, C.A.; Allen, C.M.; Akbari, A.; Collier, C.P.; Holland, D.C.; Day, A.G.; Knoll, G.A. Comparison of the new and traditional CKD-EPI GFR estimation equations with urinary inulin clearance: A study of equation performance. Clin. Chim. Acta 2019, 488, 189-195. [CrossRef]

19. Murton, M.; Goff-Leggett, D.; Bobrowska, A.; Garcia Sanchez, J.J.; James, G.; Wittbrodt, E.; Stephen Nolan, S.; Sörstadius, E.; Pecoits-Filho, R.; Tuttle, K. Burden of chronic kidney disease by KDIGO categories of glomerular filtration rate and albuminuria: A systematic review. Adv. Ther. 2021, 38, 180-200. [CrossRef]

20. Reilly, R.; Spalding, S.; Walsh, B.; Wainer, J.; Pickens, S.; Royster, M.; Villanacci, J.; Little, B.B. Chronic environmental and occupational lead exposure and kidney function among African Americans: Dallas Lead Project II. Int. J. Environ. Res. Public Health 2018, 15, 2875. [CrossRef]

21. Sommar, J.N.; Svensson, M.K.; Björ, B.M.; Elmståhl, S.I.; Hallmans, G.; Lundh, T.; Schön, S.M.; Skerfving, S.; Bergdahl, I.A. Endstage renal disease and low-level exposure to lead, cadmium and mercury; a population-based, prospective nested case-referent study in Sweden. Environ. Health 2013, 12, 9. [CrossRef] [PubMed]

22. Harari, F.; Sallsten, G.; Christensson, A.; Petkovic, M.; Hedblad, B.; Forsgard, N.; Melander, O.; Nilsson, P.M.; Borné, Y.; Engström, G.; et al. Blood lead levels and decreased kidney function in a population-based cohort. Am. J. Kidney Dis. 2018, 72, 381-389. [CrossRef] [PubMed]

23. Satarug, S. Dietary cadmium intake and its effects on kidneys. Toxics 2018, 6, 15. [CrossRef]

24. Satarug, S.; Gobe, G.C.; Vesey, D.A.; Phelps, K.R. Cadmium and lead exposure, nephrotoxicity, and mortality. Toxics 2020, 8, 86. [CrossRef]

25. Buser, M.C.; Ingber, S.Z.; Raines, N.; Fowler, D.A.; Scinicariello, F. Urinary and blood cadmium and lead and kidney function: NHANES 2007-2012. Int. J. Hyg. Environ. Health 2016, 219, 261-267. [CrossRef] [PubMed]

26. Wang, W.; Schaumberg, D.A.; Park, S.K. Cadmium and lead exposure and risk of cataract surgery in U.S. adults. Int. J. Hyg. Environ. Health 2016, 219, 850-856. [CrossRef] [PubMed]

27. Shim, Y.K.; Lewin, M.D.; Ruiz, P.; Eichner, J.E.; Mumtaz, M.M. Prevalence and associated demographic characteristics of exposure to multiple metals and their species in human populations: The United States NHANES, 2007-2012. J. Toxicol. Environ. Health A 2017, 80, 502-512. [CrossRef]

28. Jin, R.; Zhu, X.; Shrubsole, M.J.; Yu, C.; Xia, Z.; Dai, Q. Associations of renal function with urinary excretion of metals: Evidence from NHANES 2003-2012. Environ. Int. 2018, 121, 1355-1362. [CrossRef]

29. Saravanabhavan, G.; Werry, K.; Walker, M.; Haines, D.; Malowany, M.; Khoury, C. Human biomonitoring reference values for metals and trace elements in blood and urine derived from the Canadian Health Measures Survey 2007-2013. Int. J. Hyg. Environ. Health 2017, 220, 189-200. [CrossRef]

30. Liao, K.W.; Pan, W.H.; Liou, S.H.; Sun, C.W.; Huang, P.C.; Wang, S.L. Levels and temporal variations of urinary lead, cadmium, cobalt, and copper exposure in the general population of Taiwan. Environ. Sci. Pollut. Res. Int. 2019, 26, 6048-6064. [CrossRef]

31. Kim, N.S.; Ahn, J.; Lee, B.K.; Park, J.; Kim, Y. Environmental exposures to lead, mercury, and cadmium among South Korean teenagers (KNHANES 2010-2013): Body burden and risk factors. Environ. Res. 2017, 156, 468-476. [CrossRef] [PubMed]

32. Buchet, J.P.; Lauwerys, R.; Roels, H.; Bernard, A.; Bruaux, P.; Claeys, F.; Ducoffre, G.; de Plaen, P.; Staessen, J.; Amery, A.; et al. Renal effects of cadmium body burden of the general population. Lancet 1990, 336, 699-702. [CrossRef]

33. Akesson, A.; Lundh, T.; Vahter, M.; Bjellerup, P.; Lidfeldt, J.; Nerbrand, C.; Samsioe, G.; Strömberg, U.; Skerfving, S. Tubular and glomerular kidney effects in Swedish women with low environmental cadmium exposure. Environ. Health Perspect. 2005, 113, 1627-1631. [CrossRef] [PubMed]

34. Barregard, L.; Bergstrom, G.; Fagerberg, B. Cadmium, type 2 diabetes, and kidney damage in a cohort of middle-aged women. Environ. Res. 2014, 135, 311-316. [CrossRef] [PubMed]

35. Haswell-Elkins, M.; Satarug, S.; O’Rourke, P.; Moore, M.; Ng, J.; McGrath, V.; Walmby, M. Striking association between urinary cadmium level and albuminuria among Torres Strait Islander people with diabetes. Environ. Res. 2008, 106, 379-383. [CrossRef]

36. Hwangbo, Y.; Weaver, V.M.; Tellez-Plaza, M.; Guallar, E.; Lee, B.K.; Navas-Acien, A. Blood cadmium and estimated glomerular filtration rate in Korean adults. Environ. Health Perspect. 2011, 119, 1800-1805. [CrossRef]

37. Bökenkamp, A. Proteinuria-take a closer look! Pediatr. Nephrol. 2020, 35, 533-541. [CrossRef]

38. Hornung, R.W.; Reed, L.D. Estimation of average concentration in the presence of nondetectable values. Appl. Occup. Environ. Hyg. 1990, 5, 46-51. [CrossRef]

39. Flannery, B.M.; Dolan, L.C.; Hoffman-Pennesi, D.; Gavelek, A.; Jones, O.E.; Kanwal, R.; Wolpert, B.; Gensheimer, K.; Dennis, S.; Fitzpatrick, S.U.S. Food and Drug Administration's interim reference levels for dietary lead exposure in children and women of childbearing age. Regul. Toxicol. Pharmacol. 2020, 110, 104516. [CrossRef]

40. Padilla, M.A.; Elobeid, M.; Ruden, D.M.; Allison, D.B. An examination of the association of selected toxic metals with total and central obesity indices: NHANES 99-02. Int. J. Environ. Res. Public Health 2010, 7, 3332-3347. [CrossRef] 
41. Jain, R.B. Effect of pregnancy on the levels of blood cadmium, lead, and mercury for females aged 17-39 years old: Data from National Health and Nutrition Examination Survey 2003-2010. J. Toxicol. Environ. Health A 2013, 76, 58-69. [CrossRef] [PubMed]

42. Garner, R.; Levallois, P. Cadmium levels and sources of exposure among Canadian adults. Health Rep. 2016, 27, 10-18. [PubMed]

43. Akbar, L.; Zuk, A.M.; Martin, I.D.; Liberda, E.N.; Tsuji, L.J.S. Potential obesogenic effect of a complex contaminant mixture on Cree First Nations adults of Northern Québec, Canada. Environ. Res. 2021, 192, 110478. [CrossRef] [PubMed]

44. Sonthon, P.; Promthet, S.; Changsirikulchai, S.; Rangsin, R.; Thinkhamrop, B.; Rattanamongkolgul, S.; Hurst, C.P. The impact of the quality of care and other factors on progression of chronic kidney disease in Thai patients with type 2 diabetes mellitus: A nationwide cohort study. PLoS ONE 2017, 12, e0180977. [CrossRef] [PubMed]

45. Dhooge, W.; Hond, E.D.; Koppen, G.; Bruckers, L.; Nelen, V.; Van De Mieroop, E.; Bilau, M.; Croes, K.; Baeyens, W.; Schoeters, G.; et al. Internal exposure to pollutants and body size in Flemish adolescents and adults: Associations and dose-response relationships. Environ. Int. 2010, 36, 330-337. [CrossRef] [PubMed]

46. Shao, W.; Liu, Q.; He, X.; Liu, H.; Gu, A.; Jiang, Z. Association between level of urinary trace heavy metals and obesity among children aged 6-19 years: NHANES 1999-2011. Environ. Sci. Pollut. Res. Int. 2017, 24, 11573-11581. [CrossRef] [PubMed]

47. Satarug, S.; Boonprasert, K.; Gobe, G.C.; Ruenweerayut, R.; Johnson, D.W.; Na-Bangchang, K.; Vesey, D.A. Chronic exposure to cadmium is associated with a marked reduction in glomerular filtration rate. Clin. Kidney. J. 2018, 12, 468-475. [CrossRef]

48. Satarug, S.; Gobe, G.C.; Ujjin, P.; Vesey, D.A. A comparison of the nephrotoxicity of low doses of cadmium and lead. Toxics 2020, 8, 18. [CrossRef]

49. Satarug, S.; Vesey, D.A.; Ruangyuttikarn, W.; Nishijo, M.; Gobe, G.C.; Phelps, K.R. The source and pathophysiologic significance of excreted cadmium. Toxics 2019, 7, 55. [CrossRef]

50. Satarug, S.; Vesey, D.A.; Nishijo, M.; Ruangyuttikarn, W.; Gobe, G.C.; Phelps, K.R. The effect of cadmium on GFR is clarified by normalization of excretion rates to creatinine clearance. Int. J. Mol. Sci. 2021, 22, 1762. [CrossRef]

51. Filippini, T.; Wise, L.A.; Vinceti, M. Cadmium exposure and risk of diabetes and prediabetes: A systematic review and doseresponse meta-analysis. Environ. Int. 2021, 158, 106920. [CrossRef] [PubMed]

52. Gerich, J.E. Role of the kidney in normal glucose homeostasis and in the hyperglycaemia of diabetes mellitus: Therapeutic implications. Diabet. Med. 2010, 27, 136-142. [CrossRef] [PubMed]

53. DeFronzo, R.A.; Davidson, J.A.; Prato, S.D. The role of the kidneys in glucose homeostasis: A new path towards normalizing glycaemia. Diabetes Obes. Metab. 2012, 14, 5-14. [CrossRef] [PubMed]

54. Fay, M.J.; Alt, L.A.C.; Ryba, D.; Salamah, R.; Peach, R.; Papaeliou, A.; Zawadzka, S.; Weiss, A.; Patel, N.; Rahman, A.; et al Cadmium nephrotoxicity is associated with altered microRNA expression in the rat renal cortex. Toxics 2018, 6, 16. [CrossRef] [PubMed]

55. Fitzgerald, R.; Olsen, A.; Nguyen, J.; Wong, W.; El Muayed, M.; Edwards, J. Pancreatic islets accumulate cadmium in a rodent model of cadmium-induced hyperglycemia. Int. J. Mol. Sci. 2020, 22, 360. [CrossRef] [PubMed] 